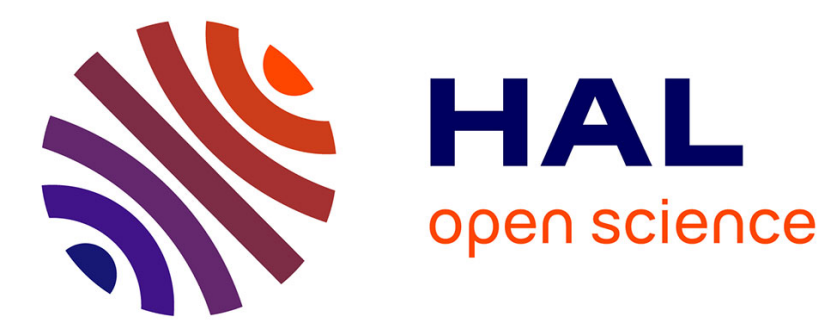

\title{
ÉTALONNAGE D'UNE MESURE OBJECTIVE D'INTELLIGIBILITÉ PAR DES TESTS SUBJECTIFS
}

\author{
L. Morellini
}

\section{To cite this version:}

L. Morellini. ÉTALONNAGE D'UNE MESURE OBJECTIVE D'INTELLIGIBILITÉ PAR DES TESTS SUBJECTIFS. Journal de Physique IV Proceedings, 1992, 02 (C1), pp.C1-339-C1-342. 10.1051/jp4:1992173 . jpa-00251245

\section{HAL Id: jpa-00251245 https://hal.science/jpa-00251245}

Submitted on 1 Jan 1992

HAL is a multi-disciplinary open access archive for the deposit and dissemination of scientific research documents, whether they are published or not. The documents may come from teaching and research institutions in France or abroad, or from public or private research centers.
L'archive ouverte pluridisciplinaire HAL, est destinée au dépôt et à la diffusion de documents scientifiques de niveau recherche, publiés ou non, émanant des établissements d'enseignement et de recherche français ou étrangers, des laboratoires publics ou privés. 


\title{
ETALONNAGE D'UNE MESURE OBJECTIVE D'INTELLIGIBILITE PAR DES TESTS SUBJECTIFS
}

\author{
L. MORELLINI \\ Laboratoire d'Electromagnétisme et d'Acoustique, Département d'Electricité, Ecole Polytechnique \\ Fédérale de Lausanne, CH-1015 Lausanne, Switzerland
}

\begin{abstract}
The aim of this research has been to calibrate an objective intelligibility measurement using subjective intelligibility data. Trials were carried out for simple cases of disturbance such as bandwidth limitations and additional noise. The subjective results obtained were compared to the results supplied by the measurement of the STI (Speech Transmission Index). This comparison led to the calibration curve, which has been plotted using two differents methods of measuring the speech level: RMS (A-weighted) and EPL (Equivalent Peak Level, A-weighted).
\end{abstract}

\section{Résumé}

Le but de cette recherche est l'étalonnage d'une mesure objective d'intelligibilité à partir d'une base de données d'intelligibilité subjective. Des essais ont été conduits pour des cas simples de perturbation tels que limitation de bande passante et bruit. Le résultat de la mesure subjective est comparé à la mesure du STI (Speech Transmission Index). Cette comparaison conduit à la courbe d'étalonnage, obtenue en utilisant deux méthodes de mesure du niveau de parole: niveau RMS (pondéré A) et EPL (Equivalent Peak Level, pondéré $A$ ).

\section{Introduction}

Cette communication présente l'étalonnage d'une mesure objective d'intelligibilité à partir d'une base de données d'intelligibilité subjective, qui fait l'objet d'un projet de recherche et développement à la demande du Groupement de l'Armement Suisse.

Ce problème concerne les télécommunications entre mobiles, par exemple les transmissions militaires, dans lesquelles les causes principales de pertes d'intelligibilité sont les bruits, les fluctuations de niveau et la limitation de largeur de bande. Pour les équipements de transmission modernes (vocoders ou autres procédés de codage de la parole, $[1,2,3]$ ) on ne dispose pas encore de données précises de corrélation entre mesures objectives et appréciation subjective de la qualité de transmission.

Nous avons entrepris une recherche visant à étalonner les mesures objectives à l'aide d'une base de données de tests subjectifs d'intelligibilité.

\section{Mesure subjective}

Dans une première phase, une Station d'Evaluation Subjective d'Intelligibilité (SESI) a été réalisée [4], pour la conduite semi-automatique de mesures subjectives [5]. La station est transportable et 
permet le test simultané avec 6 auditeurs dans des conditions de transmission déterminées, via un équipement de transmission réel ou simulé.

Un Rhyme Test avec choix fermé de la réponse est utilisé. Les stimuli sont des logatomes CVC [6], précédés d'une phrase d'introduction.

Des logiciels simplifient les tâches du responsable de la conduite du test, en minimisant les erreurs de manipulation et en garantissant une sécurité dans la gestion d'une grande quantité d'informations: à chaque test toutes les informations concernant les conditions de test, les auditeurs et toutes les réponses sont sauvegardées.

Toute séance de test d'un groupe de six auditeurs commence par un relevé automatique du seuil d'audition.

Le stimulus mélangé avec un bruit blanc, est filtré passe-bande de 300 à $3400 \mathrm{~Hz}$.

Les auditeurs disposent, pour répondre, d'un mini-terminal présentant les alternatives (maximum six) et ayant des interrupteurs en correspondance de chaque alternative. Ces alternatives de réponse sont construites et présentées aléatoirement à l'affichage, par modification de la première consonance. Deux interrupteurs supplémentaires reçoivent les réponses du type "je n'ai rien entendu" ou "plusieurs réponses sont possibles". Ces réponses permettent d'augmenter la précision du test en cas de fortes perturbation et d'envisager un traitement ultérieur en cas de doute de l'auditeur.

Pendant les tests subjectifs pratiqués jusqu'à maintenant, le rapport signal/bruit changeait par liste de 10 logatomes, selon une séquence pseudo-aléatoire, par pas de $2 \mathrm{~dB}$ entre -10 et $+10 \mathrm{~dB}$. Ces tests nous ont permis d'atteindre un niveau de confiance de $99 \%$ à $5 \%$ d'écart du score, avec 21 auditeurs naîfs et 205 listes de $10 \mathrm{CVC}$.

\section{Mesure du niveau de parole}

Les principes de conception de la station SESI nous permettent de changer de méthode d'évaluation du niveau de parole sans répéter les essais subjectifs. Cette caractéristique nous a permis d'évaluer plusieurs méthodes de mesure pour satisfaire à la nécessité d'obtenir des valeurs caractéristiques du niveau des logatomes. Les méthodes prises en considération sont les suivantes :

- RMS : valeur efficace

- $\quad$ RMS(A) : valeur efficace pondérée A

- $\quad$ EPL(seuil) : niveau de crête équivalent, le seuil est donné par rapport au niveau RMS [7]

- EPL(A,seuil) : niveau de crête équivalent pondéré A, le seuil est donné par rapport au niveau $\operatorname{RMS}(\mathrm{A})$.

Le niveau est calculé sur toute la durée du logatome.

La bảse de données stimuli (190 logatomes) utilisée lors des tests a été mesurée et les résultats montrent que pour le calcul du EPL un seuil situé à $-15 \mathrm{~dB}$ donne les écart les plus petits entre niveau mesuré et moyenne entre les niveaux mesurés pour les seuils de $-25 \mathrm{~dB}$ à $0 \mathrm{~dB}$.

Finalement, deux méthodes ont été retenues :

- RMS(A): valeur efficace pondérée A

- $\quad \operatorname{EPL}(\mathrm{A}$, seuil) : niveau de crête équivalent pondéré $\mathrm{A}$, le seuil est donné par rapport au niveau RMS(A) (le mềme seuil a été utilisé par Steeneken dans [8]).

Les mêmes méthodes de mesure ont été appliquées aux bruits.

Les logiciels développés permettent de construire des matrices de confusion entre consonances.

\section{Mesure objective}

L'équipement de mesure objective utilisé est le STIDAS II-D [9]. La même bande passante que pour la mesure subjective a été employée ici. Le STI a été mesuré pour des rapports signal/bruit de $-12 \mathrm{~dB}$ à $+25 \mathrm{~dB}$ par pas de $0.375 \mathrm{~dB}$. La courbe obtenue a été ajustée par un polynome du 3ème degré. Le filtre étant un système linéaire, la mesure n'a été effectuée que pour la seule fréquence de modulation de $2.5 \mathrm{~Hz}$. 


\section{Etalonnage}

La figure 1 montre les trois phases de l'étalonnage :

A mesure subjective, où l'on étudie un ou plusieurs paramètres perturbateurs du système de télécommunications testé (ici le rapport signal/bruit)

B mesure objective, le(s) même(s) paramètre(s) qu'en A est (sont) étudié(s)

C corrélation entre les deux mesures.

Par la suite, un système de télécommunications similaire (on entend par similaire, un système ayant les mêmes caractéristiques de bande passante, dynamique et linéarité) pourra être évalué dans des conditions d'exploitation bien précises par une mesure objective: la courbe d'étalonnage permettra de transformer le résultat objectif en un score d'intelligibilité.

Les courbes $\mathrm{C}$ de la figure 1 montrent que la mesure $\operatorname{EPL}(\mathrm{A},-15)$ conduit à une plus grande variabilité du STI.

Les accidents de la courbe sont dûs au faible nombre d'essais subjectifs. Une régression polynomiale d'ordre 3 donne une corrélation de 0.959 entre STI et RMS(A) et 0.982 entre STI et EPL $(A,-15)$.

La différence des deux courbes s'explique par une différence plus grande des niveaux EPL(A,-15) RMS(A) pour la parole que pour le bruit. Cette différence cause un basculement vers la droite de la courbe d'étalonnage et un réarrangement des niveaux dû aux différences des niveaux $\operatorname{EPL}(A,-15)$ RMS(A) entre logatomes.

Malgré le nombre d'auditeurs et de tests encore faibles, la mesure subjective semi-automatique démontre une précision assez remarquable.

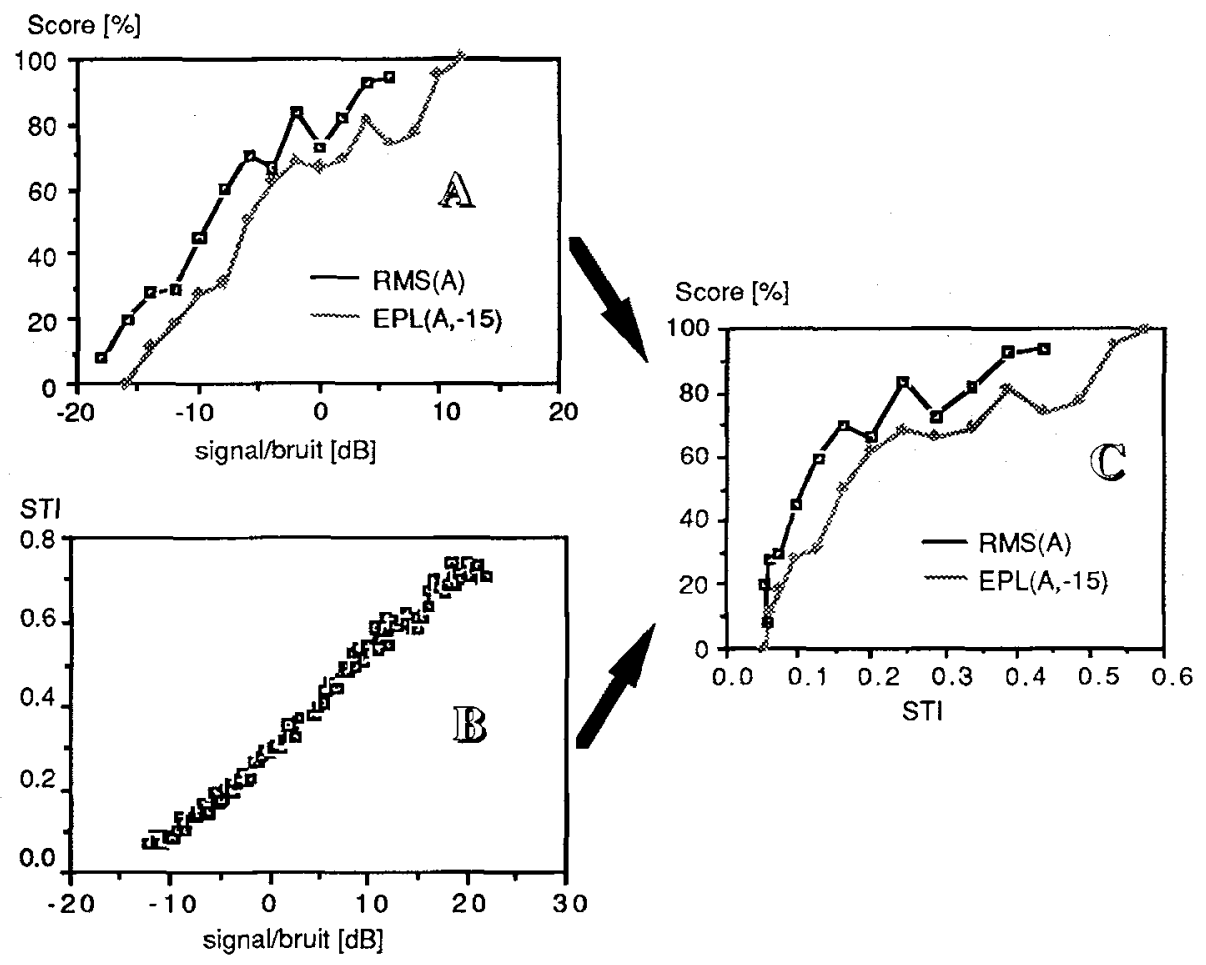

Figure 1 Phases de l'étalonnage: A - mesure subjective, B - mesure objective, C - élimination du paramètre rapport signal/bruit 


\section{Poursuite des travaux}

Actuellement ces mesures continuent pour augmenter la précision de la courbe d'étalonnage.

En [6], les logatomes sont composés à partir de listes de consonances, formées d'une à deux consonnes. L'étude des matrices de confusion a montré la tendance à reconnaître, en cas de consonance "double", une des deux consonnes. Un nombre de tests plus important et la possibilité de choisir en alternative de réponse une consonance ayant le même nombre de consonnes, nous permettront de mieux interpréter ce score.

Nous avons aussi établi un protocole permettant d'obtenir des scores d'intelligibilité subjective pour des mesures sur des systèmes de télécommunications en conditions d'utilisation et de perturbations réelles.

Des mesures sont planifiées sur des systèmes de télécommunications utilisant des appareils de chiffrage de la parole, lesquels introduisent une forte limitation de bande passante et des non linéarités (expansion/compression).

\section{Bibliographie}

[1] M. Aoki, Fumiaki Ishito, Fukuya Ishito Speech Quality of Conversational Packetized Voice Communication The Transaction of the IECE of Japan, Vol E69, No. 2, février 1986, pp. 107-112.

[2] D. J. Goodman, J. S. Goodman, M. Chen Intelligibility and Rating of Digitally Coded Speech IEEE 1978, pp. 403-409.

[3] D. Reichert Messung der Übertragungsqualität an PCM-Systemen für Sprachübertragung Fernmeldetechnik 17, 1977, H 2, pp. 62-67.

[4] F. Kehlstadt, L. Morellini, E. J. Rathe, M. Rossi Multichannel approach for speech intelligibility assessment 13 ème ICA, Belgrade 1989, Vol 2, pp. 449-452.

[5] F. Kehlstadt, L. Morellini Tests semi-automatiques de l'intelligibilité de parole dans les systèmes de transmission AGEN Mitteilungen Nr. 52, nov 1990, Berne/Suisse, pp. 53-57.

[6] J. M. Person Essais de netteté pratiqués par l'administration française des PTT FASE, Liège 1971, pp. $277-283$.

[7] P. T. Brady Equivalent Peak Level: a Threshold-Independent Speech-Level Measure J.A.S.A. vol 44 1968, pp. 695-699.

[8] H. J. M. Steeneken Comparisons among three subjective and one objective intelligibility test Rapport no IZF 1987-8, 33 pages, TNO institute of perception, Soesterberg, the Netherlands

[9] H. J. M. Steeneken, E. Agterhuis Description of STIDAS II-D Part I: general system and program description Rapport no IZF 1982-29, 27 pages, , TNO institute of perception, Soesterberg, the Netherlands 